STRUCTURAL BIOLOGY

ISSN 2059-7983

Received 8 March 2018

Accepted 12 April 2018

Edited by R. J. Read, University of Cambridge, England

₹ Current address: Instituto de Biología Molecular y Celular de Rosario (IBR, CONICETUNR), Ocampo y Esmeralda, Predio CCT, 2000 Rosario, Argentina.

Keywords: molecular replacement pipeline; SIMBAD; contaminant; lattice search; structure solution.

PDB references: Escherichia coli DPS, 6b0d; E. coli catalase HPII, 6by0; Serratia

proteamaculans cyanase, $6 \mathrm{~b} 6 \mathrm{~m}$

Supporting information: this article has supporting information at journals.iucr.org/d

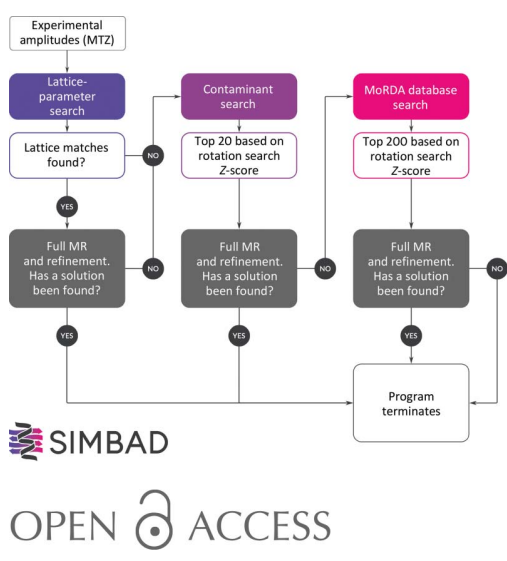

\section{SIMBAD: a sequence-independent molecular- replacement pipeline}

\author{
Adam J. Simpkin, ${ }^{\mathrm{a}, \mathrm{b}}$ Felix Simkovic, ${ }^{\mathrm{a}}$ Jens M. H. Thomas, ${ }^{\mathrm{a}}$ Martin Savko, ${ }^{\mathrm{b}}$ Andrey \\ Lebedev, ${ }^{\text {c,d }}$ Ville Uski, ${ }^{c, d}$ Charles Ballard, ${ }^{c, d}$ Marcin Wojdyr, ${ }^{c, d, e}$ Rui Wu, ${ }^{\text {, }}$ Ruslan \\ Sanishvili, ${ }^{\mathrm{g}}$ Yibin Xu, ${ }^{\mathrm{h}, \mathrm{i}}$ María-Natalia Lisa, ${ }^{\ddagger} \ddagger$ Alejandro Buschiazzo, ${ }^{\mathrm{j}}$ William \\ Shepard, ${ }^{b}$ Daniel J. Rigden ${ }^{\mathrm{a} *}$ and Ronan M. Keegan ${ }^{\mathrm{a}, \mathrm{c}, \mathrm{d}}$ *
}

\begin{abstract}
aInstitute of Integrative Biology, University of Liverpool, Liverpool L69 7ZB, England, ${ }^{\mathbf{b}}$ Synchrotron SOLEIL, L'Orme des

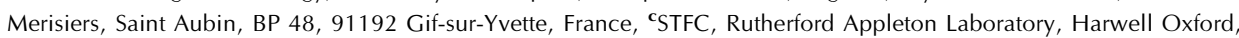
Didcot OX11 OFA, England, 'CCP4, Research Complex at Harwell, Rutherford Appleton Laboratory, Harwell Oxford, Didcot OX11 OFA, England, ${ }^{\mathbf{e}}$ Global Phasing Ltd, Cambridge CB3 OAX, England, ${ }^{\mathrm{f}}$ Feil Family Brain and Mind Institute, Weill Cornell Medicine, New York, NY 10021, USA, ${ }^{\mathrm{g}} \mathrm{GM} / \mathrm{CA} @ A P S$, The X-Ray Science Division, The Advanced Photon Source, Argonne National Laboratory, Lemont, IL 60439, USA, hDivision of Structural Biology, Walter and Eliza Hall Institute of Medical Research, Parkville, VIC 3052, Australia, 'Department of Medical Biology, University of Melbourne, Royal Parade, Parkville, VIC 3050, Australia, and 'Laboratory of Molecular and Structural Microbiology, Institut Pasteur de Montevideo, Mataojo 2020, 11400 Montevideo, Uruguay. *Correspondence e-mail: drigden@liverpool.ac.uk, ronan.keegan@stfc.ac.uk
\end{abstract}

The conventional approach to finding structurally similar search models for use in molecular replacement (MR) is to use the sequence of the target to search against those of a set of known structures. Sequence similarity often correlates with structure similarity. Given sufficient similarity, a known structure correctly positioned in the target cell by the MR process can provide an approximation to the unknown phases of the target. An alternative approach to identifying homologous structures suitable for MR is to exploit the measured data directly, comparing the lattice parameters or the experimentally derived structure-factor amplitudes with those of known structures. Here, $S I M B A D$, a new sequenceindependent MR pipeline which implements these approaches, is presented. $S I M B A D$ can identify cases of contaminant crystallization and other mishaps such as mistaken identity (swapped crystallization trays), as well as solving unsequenced targets and providing a brute-force approach where sequencedependent search-model identification may be nontrivial, for example because of conformational diversity among identifiable homologues. The program implements a three-step pipeline to efficiently identify a suitable search model in a database of known structures. The first step performs a lattice-parameter search against the entire Protein Data Bank (PDB), rapidly determining whether or not a homologue exists in the same crystal form. The second step is designed to screen the target data for the presence of a crystallized contaminant, a not uncommon occurrence in macromolecular crystallography. Solving structures with MR in such cases can remain problematic for many years, since the search models, which are assumed to be similar to the structure of interest, are not necessarily related to the structures that have actually crystallized. To cater for this eventuality, $S I M B A D$ rapidly screens the data against a database of known contaminant structures. Where the first two steps fail to yield a solution, a final step in $S I M B A D$ can be invoked to perform a brute-force search of a nonredundant PDB database provided by the MoRDa MR software. Through early-access usage of $\operatorname{SIMBAD}$, this approach has solved novel cases that have otherwise proved difficult to solve.

\section{Introduction}

In X-ray crystallography, the problem of solving the threedimensional structure of a protein remains a difficult task. Even with crystals diffracting to high resolution, many projects flounder owing to the challenges involved in overcoming the phase problem. For macromolecules with more than a few 
hundred atoms, solving the phase problem directly is currently not viable, so an alternative approach must be used. Molecular replacement (MR) is the most popular route to solve the problem as it is quick, inexpensive and can be highly automated (Evans \& McCoy, 2008; Long et al., 2008). MR exploits the fact that proteins with similar amino-acid sequences typically form similar three-dimensional structures. Where a known structure has a similar sequence to a target, the phase information from the known structure can, assuming that there is corresponding structural similarity, often be used as a starting point for the phases of the unknown structure. The procedure requires that the known structure is reorientated and positioned correctly in the unit cell of the target. Programs incorporating sophisticated scoring systems such as Phaser (McCoy et al., 2007) and MOLREP (Vagin \& Teplyakov, 2010) have been developed to perform this task. However, the selection of an appropriate search model remains a limiting factor in MR. Sequence similarity does not always ensure structural similarity, particularly where the similarity is lower than 30\% (Krissinel \& Henrick, 2004; Krissinel, 2007). Some recent studies have sought alternative ways of finding structurally similar search models. Approximating target structures through $a b$ initio modelling and using these as search models has been shown to work by Qian et al. (2007) and Rigden et al. (2008) and can be exploited using the AMPLE application (Bibby et al., 2012). Other approaches make use of idealized fragments or regularly occurring fragments and motifs from known structures as search models in MR. ARCIMBOLDO (Rodríguez et al., 2009) and Fragon (Jenkins, 2018) are two developments exploiting this approach. All of these applications mainly rely on small but highly accurate fragments being placed correctly in the unit cell of the target. In the most extreme cases, where data are available to $1 \AA$ resolution or better, it has been shown that it is possible to use single atoms as a successful search model (McCoy et al., 2017).

For the more traditional sequence-based approach, much effort has been put into developing software pipelines that will attempt to find a solution from a large set of carefully crafted search models from potentially suitable homologues. Examples of these include $M o R D a$ (Vagin \& Lebedev, 2015), MrBUMP (Keegan et al., 2018), BALBES (Long et al., 2008) and MRage (McCoy et al., 2007). The search models selected by these applications or manually by a user can give poor results for a number of reasons. These include insensitivity of the template search (i.e. the homologue is too divergent from the actual structure), misleading sequence information (i.e. a contaminant has been crystallized in place of the desired protein) or the sequence similarity providing an imperfect proxy for structural similarity (i.e. where relatives with high sequence similarity have been crystallized in different conformational states). In such cases, ARCIMBOLDO and Fragon may retrieve the solution through the correct placement of idealized fragments such as helices, but are limited by the resolution requirements of SHELXE $(\sim 2.4 \AA$; Thorn \& Sheldrick, 2013) and ACORN ( 1.7 Å; Foadi et al., 2000; Yao et al., 2005), respectively, when improving upon the phases given by the initial placement of the fragment by Phaser. Some developments have sought to overcome these problems by attempting to unearth suitable search models through a bruteforce search of the PDB (Stokes-Rees \& Sliz, 2010; Hatti et al., 2016). ContaMiner (Hungler et al., 2016) is another approach specifically aimed at finding contaminants by testing a library of known contaminants in MR.

Here, we present a new pipeline, SIMBAD (SequenceIndependent Molecular replacement Based on Available Databases), which can be used for both contaminant and brute-force approaches. Its ability to detect contaminant crystal structures is relevant to cases such as Keegan et al. (2016), where the structure remained unsolved for 14 years. It ensures acceptably low run times by testing only the nonredundant PDB entries as defined in the MoRDa database and shortcutting the process by testing first for crystals with a familiar unit cell or containing known contaminants. $M o R D a$ is a conventional MR pipeline built upon the MOLREP program. Its database contains chains from a redundancyremoved version of the PDB database and definitions of how to construct domains, oligomers, complexes and ensembles from the individual chains. In its current implementation, $S I M B A D$ uses only the domain definitions to create search models. In total, SIMBAD contains three steps: a latticeparameter search, a contaminant search and the nonredundant PDB MoRDa database search (henceforth referred to as the MoRDa DB search). Each can be run as a separate module, with the complete run involving all three steps being referred to as the combined search.

In the absence of relevant sequence-identity information to help isolate and score suitable search models, SIMBAD makes use of the rotation-function step in MR to rank search models ahead of performing a full MR search. The rotation function is a three-dimensional search used to determine the proper orientation of a search model. It was first discussed in the context of the self-Patterson by Hoppe (1957) and Huber (1965). However, the rotation function that we know today was first proposed by Rossmann \& Blow (1962). This initial rotation function exploited noncrystallographic symmetry to recover the phases required for structure determination. Rossmann and Blow also recognized that this concept could be applied to the problem of positioning a known molecule in an unknown crystal lattice by applying an additional translation procedure. The rotation search was first applied in this context by Crowther \& Blow (1967). The original rotation function was a slow calculation. Crowther expanded the Patterson functions in terms of spherical harmonics and spherical Bessel functions to create the fast rotation function (Crowther, 1972). Navaza further refined the fast rotation function to use a numerical integration rule in place of expansions in the radial function (Navaza, 1987). It was this version of the rotation function that was incorporated into AMoRe (Navaza, 1993).

More recently, Read began exploring maximum-likelihood methods as an alternative way to approach the rotation function (Read, 2001). An initial implementation added to Beast (Read, 1999, 2001) demonstrated an increase in sensitivity compared with Patterson-based rotation functions when 
applied to difficult cases. This initial maximum-likelihood method was a slow calculation. Storoni and coworkers introduced a likelihood-enhanced fast rotation function for implementation in Phaser (Storoni et al., 2004). The likelihood-enhanced fast rotation function utilizes series approximations to the full likelihood target that can be calculated quickly by the fast Fourier transform. This approximation of the full likelihood target improves the speed by several orders of magnitude. More recently, Caliandro and coworkers developed a probabilistic approach to the rotation problem in RENO09 (Caliandro et al., 2009). Similarly to the maximum-likelihood methods already discussed, the probabilistic approach constructs probability distributions for a rotated model in a given environment, although the final formulas derived differ from those obtained via maximumlikelihood principles.

SIMBAD performs the rotation search $\sim 90000$ times when screening the full MoRDa DB and, as such, speed and efficiency are very important. In light of this, the $A M o R e$ rotation function was selected, as the modular nature of the program allowed us to pre-calculate a spherical harmonic coefficient database from the 90000 models, a prerequisite for the rotation search. Ultimately this approach was not adopted, but it was the initial motivation behind the selection of AMoRe. However, the speed of the AMoRe rotation function (of the order of seconds) made the processing of such large numbers of search models tractable on a modest cluster.

In all cases the best matches are tested by MR and refinement to ascertain whether or not they give a solution. $S I M B A D$ can make use of multi-core clusters to speed up its processing of search models, enabling its combined three-step functionality to be run, for example, in the space of a few hours on a 100-core machine (2.8 GHz, AMD Opteron 4184). The software is distributed with the CCP4 suite (Winn et al., 2011) and will be made available through the CCP4 online/ cloud developments in the future. It can also be run as part of the data-processing pipelines at synchrotron beamlines to test for the presence of contaminants early in the structure-solution process.

\section{Methodology}

\subsection{Strategy}

A flowchart of the $S I M B A D$ pipeline is presented in Fig. 1. Within the three-step procedure of SIMBAD, two different methods are used to identify unknown crystals independently of sequence. The first method searches for structures in the PDB with similar lattice parameters to the unknown structure. Similar lattice parameters often indicate that a different, previously characterized protein has been crystallized by mistake (Niedzialkowska et al., 2016). The second method exploits the AMoRe (Navaza, 1994) rotation search to screen a database of candidate search models. This is split into two steps. The first step consists of screening a small database of structures that have been identified to commonly contaminate crystals. The second step consists of screening the full MoRDa
DB. The $M o R D a$ DB run is by far the most computationally expensive step and therefore the lattice-parameter/contaminant searches are run first.

\subsection{Lattice-parameter search}

The SIMBAD lattice-parameter search employs a similar strategy to that used by the Nearest-cell server (Ramraj et al., 2012) and the SAUC server (McGill et al., 2014). A database was created from the PDB containing the Niggli reduced cell, a reduced $P 1$ cell (Andrews \& Bernstein, 2014), for each structure using the explore_metric_symmetry routine in cctbx (Computational Crystallography Toolbox; https:// github.com/cctbx/cctbx_project). The Niggli reduced cell for the unknown data set is generated in the same way and compared with the Niggli reduced cells in the database.

The comparison takes place in two steps. Firstly, the Niggli reduced-cell database is searched for cells where each lattice parameter is within $\pm 5 \%$ of the respective lattice parameter in the experimental data. Secondly, a penalty score is generated for each Niggli reduced cell using

$$
\begin{aligned}
\text { penalty }= & \left|\left(a_{\mathrm{e}}-a_{\mathrm{d}}\right)\right|+\left|\left(b_{\mathrm{e}}-b_{\mathrm{d}}\right)\right|+\left|\left(c_{\mathrm{e}}-c_{\mathrm{d}}\right)\right|+\left|\left(\alpha_{\mathrm{e}}-\alpha_{\mathrm{d}}\right)\right| \\
& +\left|\left(\beta_{e}-\beta_{\mathrm{d}}\right)\right|+\left|\left(\gamma_{\mathrm{e}}-\gamma_{\mathrm{d}}\right)\right|,
\end{aligned}
$$

where $a, b$ and $c$ represent the lengths of the cell edges and $\alpha$, $\beta$ and $\gamma$ represent the angles between them. A subscript $\mathrm{e}$ signifies experimentally derived lattice parameters and a subscript $d$ is used for Niggli reduced-cell database-derived lattice parameters.

To test the intuition that a lower penalty score would be more likely to lead to a solution, a test set of 125 data sets were randomly selected from the PDB (Supplementary Table S1).

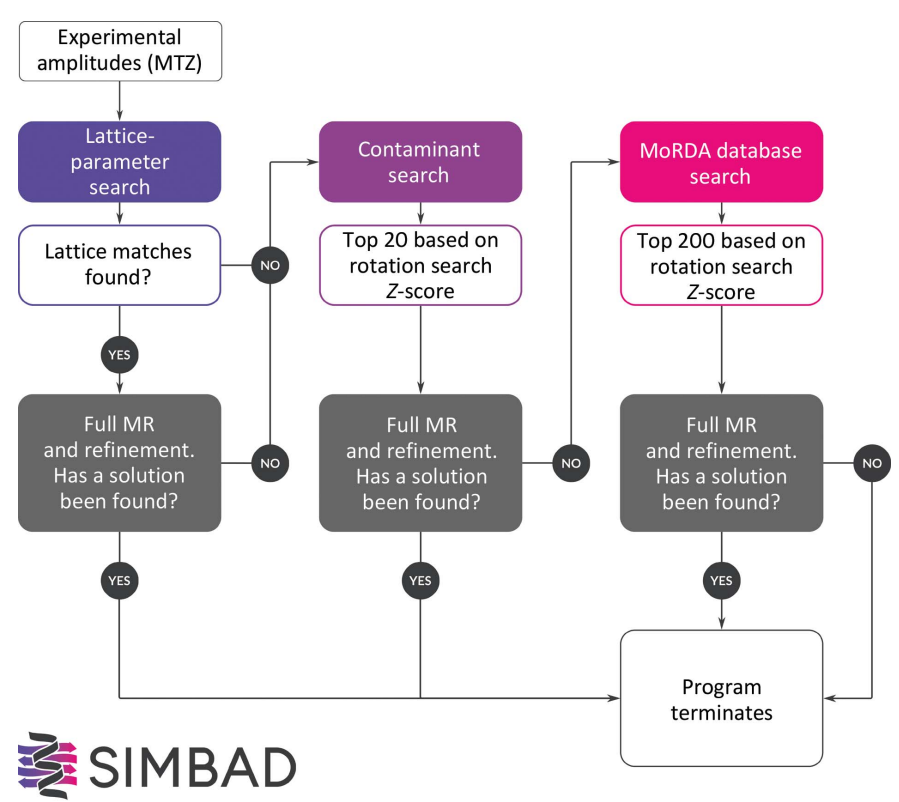

Figure 1

Flowchart detailing the decision processes in the SIMBAD pipeline. The Full MR step in each case refers to performing a complete MR procedure (rotation and translation search) using the best-ranked models from the initial search (lattice-parameter, contaminant or MoRDa DB). 
By performing the lattice-parameter search on each of these data sets, a total of 2009 unique candidates with varying penalty scores were obtained. For each candidate, MR and refinement were carried out against the relevant data set using MOLREP and REFMAC5 (Murshudov et al., 2011). A search model/penalty score was considered to have given a solution if the $R_{\text {free }}$ fell below 0.45 . These data were used to train a logistic regression classifier (Fig. 2). The training was used to fit a sigmoid function to the data, giving the equation

$$
\text { probability }=\frac{1}{1+\exp [-(-1.01 \times \text { penalty }+2.11)]} .
$$

The accuracy with which the model predicted whether a candidate search model would lead to success in MR was evaluated at $87 \%$ on the test set, matching the $87 \%$ on the training set (Supplementary Table S2). This model has been implemented into SIMBAD to give users an indication of whether a candidate is likely to return a solution.

Our model suggests that below a penalty score of 2.1 the probability of finding a solution exceeds $50 \%$. In our data set, not a single example was found where a penalty score above 12 returned a solution. Therefore, the lattice-parameter search was set to return up to 50 models with penalty scores below 12 by default.

\subsection{Rotational search}

SIMBAD uses the $A M o R e$ fast rotation function to screen databases for suitable MR candidates. By skipping models estimated to be unable to fit into the unit cell (by requiring a solvent content above $30 \%$ ) and by exploiting coarse-grained parallelization across a multi-CPU cluster, the time required for the rotation function is minimized. SIMBAD uses the correlation coefficient between the observed amplitudes for the crystal and the calculated amplitudes for the model (CC_F) to score the results from AMoRe. A large peak in the CC_F score for the top-ranked solution is indicative of a correctly orientated structure. Therefore, in order to compare the solutions for each template structure used, AMoRe was modified to return a $Z$-score of the CC_F scores. The $A M o R e$ $R O T N D O$ subroutine was modified to output $Z$-scores derived from CC_F and the correlation map. The CC_F-based $Z$-score estimates the mean and variance for the template using 200 random orientations.

2.3.1. Contaminant search. A set of 349 structures representing the different homologues and space groups of 60 proteins known to commonly occur as contaminants has been compiled. This set consists of contaminants identified in the course of developing SIMBAD and common contaminants listed by other sources (Niedzialkowska et al., 2016; Hungler $e t$ al., 2016). In addition, corresponding domains from the $M o R D a$ DB which may form subcomponents of the contaminants augment the original database. The complete list is processed in the $A M o R e$ rotation search and the models are ranked by $Z$-score. The top 20 are passed on to MOLREP and REFMAC5 for full MR and refinement.
2.3.2. MoRDa DB search. The MoRDa DB step of $S I M B A D$ screens the $M o R D a$ DB for potential MR templates. $M o R D a$ includes its own edited version of the PDB which contains a nonredundant domain database of $\sim 90000$ domains (at the time of this study). The SIMBAD pipeline processes the entire set using the fast $A M o R e$ rotation search. The models are used as they are defined in the MoRDa database with no additional modifications. Each is then ranked by $Z$-score and the top 200 solutions are passed on to MOLREP followed by REFMAC5 to perform full MR and refinement. Based on preliminary testing, this figure of 200

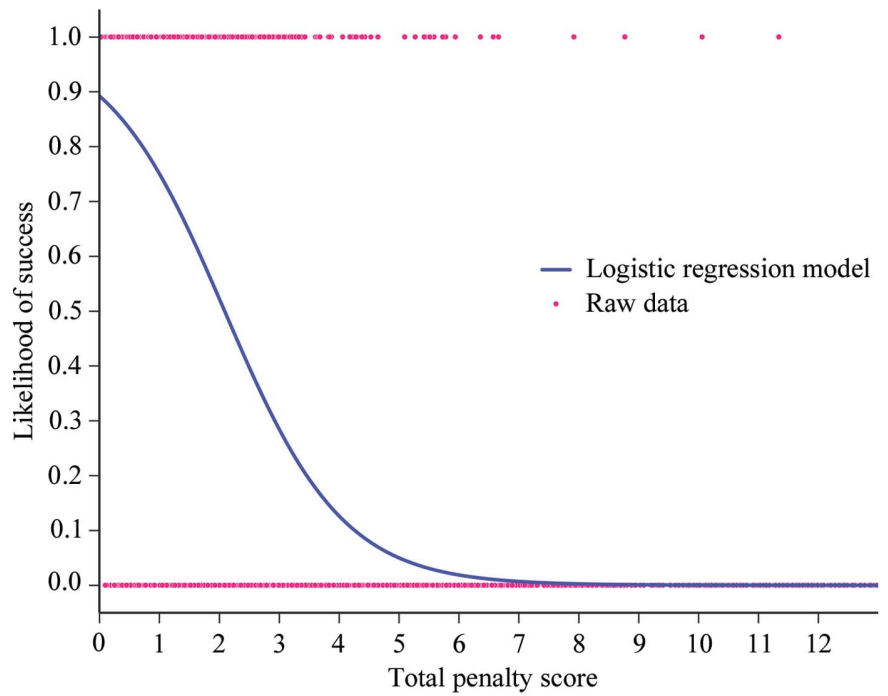

(a)

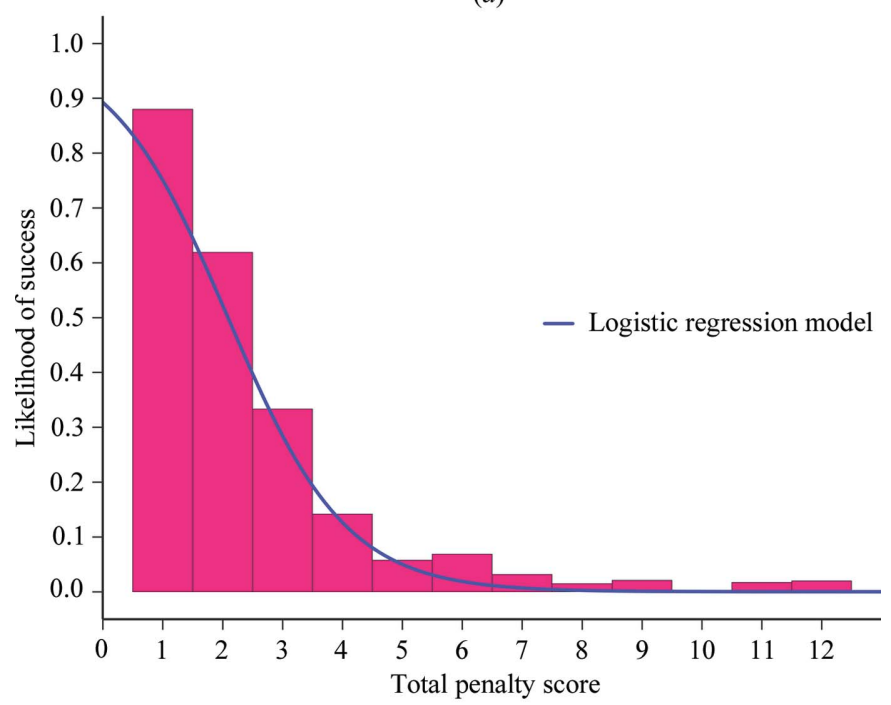

(b)

Figure 2

Logistic regression results showing the likelihood that a penalty score would result in successful MR. The purple line describing the distribution was fitted using a sigmoid model. The coefficient and intercept were determined by the 'LogisticRegression' module in sklearn (http:// www.scikit-learn.org). (a) The scatter points represent the 2009 raw data points, where the $x$ value corresponds to the total penalty score and the $y$ value is set to 1 or 0 to indicate success or failure in MR. (b) The histogram represents the proportion of success/failure for bin sizes of 1 . The figure has been truncated to show the results up to a penalty score of 13; however, the sigmoid model was calculated from data sets with penalty scores of up to 26 . 
was able to catch some nontrivial cases. Subsequent work showed it to strike a good balance between speed and sensitivity, although it has not been extensively tested.

\subsection{Full MR and refinement}

The final step in each of the lattice-parameter, contaminant and $M o R D a$ DB searches is to process the best scoring matches using first MOLREP to perform a full MR search and then REFMAC5 to refine the resulting positioned model. By default, REFMAC5 performs 30 cycles of restrained refinement for the lattice-parameter and contaminant searches and 100 cycles of restrained refinement for the MoRDa DB search. Defaults are used for all other parameters in both programs. The results are presented to the user via jsrview (Krissinel et al., 2018), a report-generating tool distributed with $C C P 4$. Tables of scores and plots of $R / R_{\text {free }}$ statistics sorted by the final $R_{\text {free }}$ value after refinement are presented to the user. An $R_{\text {free }}$ of 0.45 is suggested as indicative of a solution, but the user may also examine maps and positioned models. When $S I M B A D$ is run locally this can be performed using Coot (Emsley et al., 2010). When executed online, the moleculargraphics tool UglyMol (https://github.com/uglymol) is used instead. The $Z$-scores from the $A M o R e$ rotation search for the contaminant and MoRDa DB stages are also made available. Supplementary Fig. S1 shows the report page for a run of SIMBAD.

\section{Results}

\subsection{Testing the SIMBAD pipeline}

The first two steps of SIMBAD, the lattice-parameter and contaminant searches, are quick but thorough approaches to find search models that are suitable for MR in cases where a contaminant is present or when a related structure with very similar cell dimensions is available. Invoking these two options on their own is well suited for use as a post-data-collection rapid screening of data sets to ensure that a contaminant is not present. The follow-on step of screening the entire MoRDa DB for possible search models can, in addition to finding cases of new contaminants or misidentification, offer the possibility of finding a non-obvious search model for a novel target structure.

To realistically evaluate the capabilities of $\operatorname{SIMBAD\text {,we}}$ conducted two sets of tests. Firstly, we tested its ability to find contaminants through its lattice-parameter and contaminant searches. A second set of tests was designed to investigate how readily it can find a suitable search model from the MoRDa DB for use in determining the solution of a novel structure.

3.1.1. Testing for contaminant structure solution. The two main routes to identify the presence of a known contaminant are through the lattice-parameter search or, where this fails, through explicitly testing each entry in our contaminant list via the AMoRe rotational search. The former has the advantage of speed but relies on the contaminant crystallizing in an almost identical unit cell. The latter is more thorough but takes longer. Test results for the lattice-parameter search on simulated novel structures are given in the following section. Here, we present the results of testing the contaminant search.

In order to simulate a scenario in which a contaminant had been crystallized in a new space group/unit cell, ten structures were selected that represented a unique space group among a subset of homologues in our contaminant list. These structures were removed from our database to determine whether the contaminant search would be successful in identifying homologues in other space groups as suitable candidates for MR search models. The ten cases represented a broad range of space groups, resolutions and structure types.

SIMBAD was successful in nine out of the ten test cases (Supplementary Table S3). Analysis of the failed case (PDB entry 3fwe, an apo D138L CAP mutant) showed that the homologues for this structure had significantly larger conformational differences than for the nine successful cases. The conformational differences were measured using the pairwise structural alignment feature in GESAMT (Krissinel, 2012). The best search models were compared with the targets in terms of a $\mathrm{C}^{\alpha}$ r.m.s.d. and a $Q$-score. For the nine cases that succeeded the average $\mathrm{C}^{\alpha}$ r.m.s.d. and $Q$-score were 0.51 and 0.89 , respectively, and for the one case that failed the closest match in the contaminant database (PDB entry 3 hif) only gave a $\mathrm{C}^{\alpha}$ r.m.s.d. and $Q$-score of 1.56 and 0.75 , respectively. This model was ranked $172 \mathrm{nd}$, with a $Z$-score of 3.2. It has been shown that apo wild-type CAP (PDB entry 3hif) undergoes large conformational changes in order to bind DNA (Sharma et al., 2009). Such conformational changes would explain the intramolecular differences seen between the apo D138L CAP mutant (PDB entry 3fwe) and apo wild-type CAP (PDB entry 3hif) (Fig. 3).

In conclusion, SIMBAD is able to identify contaminants that are crystallized in a similar unit cell to existing structures using the lattice-parameter search but is also able to identify contaminants crystallized in novel ways when a sufficiently similar $\left(\mathrm{C}^{\alpha}\right.$ r.m.s.d. $\left.<1 \AA\right)$ structure is contained within our contaminant database.

3.1.2. Testing for novel structure solution. To simulate cases where the sequence is potentially unknown for a given target, we tested the SIMBAD combined search (latticeparameter, contaminant and MoRDa DB searches) against a set of 25 recently released structures in the PDB. These cases were all released in February or March 2017. The SIMBAD lattice database and the version of the MoRDa DB used at the time of testing did not contain any entries with information derived from this set of PDB structures or any subsequently released PDB entries. Other than this criterion, no particular constraints were placed on the PDB entries chosen. The set contained a wide range of resolution limits, numbers of copies in the asymmetric unit, space groups, monomer sizes and secondary-structure types (Supplementary Table S4). It also included cases that were originally solved by MR, SAD, MAD and SIRAS methods. The results of the testing are presented in Supplementary Table S4. SIMBAD was successful in 13 of the 25 test cases, a success rate of $52 \%$. Solutions were verified by a map correlation coefficient (map CC) with an electrondensity map generated for the deposited data using 


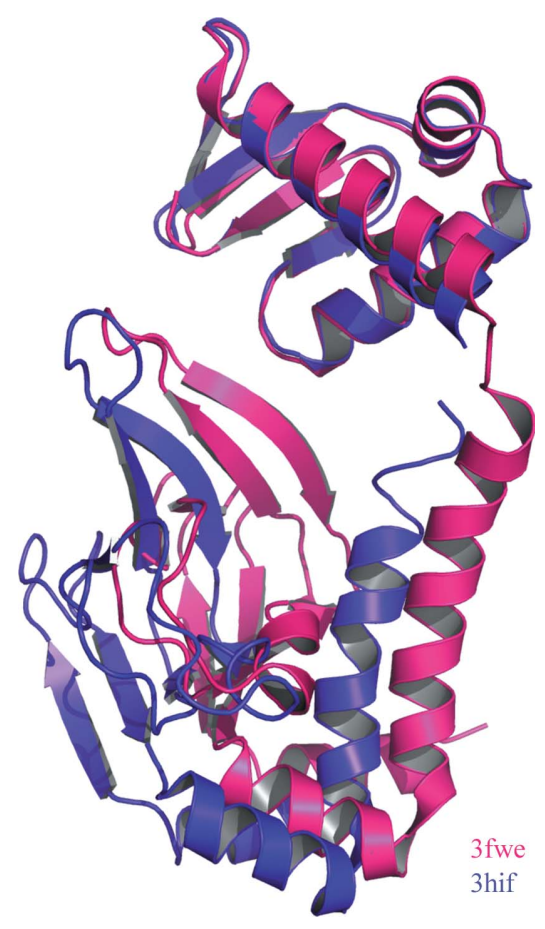

Figure 3

Structural alignment of the C-terminal DNA-binding domains of the apo D138L CAP mutant (PDB entry 3 fwe) chain $B$ (pink) and apo wild-type CAP (PDB entry 3 hif) chain $B$ (purple), highlighting the conformational change.

phenix.get_cc_mtz_mtz (Adams et al., 2010). Correct solutions had a mean map CC of 0.88 . Six cases were solved by the lattice-parameter search, with the remaining seven being solved by the MoRDa DB search.

One of the goals of our tests was to examine the degree of similarity between the model and target that was required in order to produce a solution. To this end, we examined the similarity between the top-scoring successful search model and its respective target in three different ways for each of the 25 cases. Firstly, we looked at the sequence identity. The mean sequence identity of a successful search model to the target was $98 \%$ in the lattice-parameter search and $83 \%$ in the MoRDa DB search. The lowest sequence identity between a successful search model and the target was 44\% [PDB entry 5grh using search model 3blvA_1 (MoRDa DB format: PDB code 3 blv, chain $A$, domain 1)]. We then examined the coverage of the target structure by the search model. The search model with the smallest relative size to the target was $3 j w n H \_2$, making up approximately $14 \%$ of the overall content of the asymmetric unit of PDB entry 5jqi (eight chains, 1157 residues in total). This model ranked top in the MoRDa DB search and had $100 \%$ sequence identity to the part of the target matched. On average, a successful search model made up $44 \%$ of the content of the asymmetric unit of the target. Finally, by utilizing the pairwise structural alignment feature in GESAMT, we compared the search models with the targets in terms of a $\mathrm{C}^{\alpha}$ r.m.s.d. and a $Q$-score (a measure of structural similarity, where 1 is identical and 0 is structurally unrelated). Results for successful solutions showed an average $\mathrm{C}^{\alpha}$ r.m.s.d.s and $Q$-scores of 0.63 and 0.93 , respectively, for the lattice- parameter search and 0.61 and 0.46 , respectively, for the MoRDa DB search. The highest $\mathrm{C}^{\alpha}$ r.m.s.d. between the model and the target for a success was $0.88 \AA$ (PDB entry 5mg1) in the lattice-parameter search and $1.08 \AA$ (PDB entry 5grh) in the MoRDa DB search. The MoRDa DB search ranked this model 35th, with a $Z$-score of 5.6.

In conclusion, within our test set SIMBAD was capable of producing MR solutions using search models that are significantly different from the target in terms of sequence identity $(\geq 44 \%)$, model coverage $(\geq 14 \%)$ and $C^{\alpha}$ r.m.s.d. $(\leq 1.07 \AA)$. This demonstrates the usefulness of SIMBAD for more than just known contaminant detection, showing it to be capable of finding solutions to novel structures where some search model is available with characteristics within the thresholds outlined above and possibly beyond. Notably, the resolution of the experimental data did not influence the ability to find a solution. Successful cases had resolutions in the range 1.5$3.3 \AA$.

As a follow-on to the above examination, we looked at the ability of $S I M B A D$ to pick out a possible search model from the MoRDa DB given the availability of a structure within a $\mathrm{C}^{\alpha}$ r.m.s.d. threshold of $1.07 \AA$. A GESAMT archive search of the MoRDa DB revealed that SIMBAD failed in only four of the 17 cases where there is some structure in the MoRDa DB that is within a $1.07 \AA \mathrm{C}^{\alpha}$ r.m.s.d. of the target structure (assuming a minimum alignment to $30 \%$ of the target). Of the four cases that did not produce a solution, three (PDB entries 5lnl, 5jfm and 5ayl) were multi-chain or multi-domain targets of at least seven domains. The MoRDa models most closely matching these targets provided too small a signal for them to be found in the AMoRe rotation-search step. The remaining case (PDB entry 5hsm) had a single chain of 131 residues and the best MoRDa model (3fm5A_1, C ${ }^{\alpha}$ r.m.s.d. = $0.97 \AA$ ) failed to produce a solution in SIMBAD. This model provided a weak signal in the rotation search $(Z=4)$ and was relegated to a low overall ranking by many similar, but higher scoring search models containing longer $\alpha$-helices. In the cases where a successful solution was found using the MoRDa DB search, the resulting best search model was ranked top on three occasions. The lowest $A M o R e$ ranking for a successful search model was 170 . With this step trialling more than 90000 search models, it demonstrates the sensitivity of the $Z$-scoring added to $A M o R e$ but also the value of taking at least the top 200 ranked hits to the full MR and refinement stage. The $Z$-score values for successful solutions ranged from 5.5 (PDB entry 5uqf) to 14.0 (PDB entry 5uca) with a mean of 8.9.

Finally, we looked at the run times for the various test cases. The average run time for success during the lattice-parameter step was $0.7 \mathrm{~h}$ on a maximum of 20 cores $(2.8 \mathrm{GHz}$, AMD Opteron 4184). Completion of the combined search required an average of $11.6 \mathrm{~h}$ on 40 cores, regardless of success or failure.

\subsection{User cases}

In this section, we present three cases in which SIMBAD has been used to determine a difficult-to-solve case owing to 
the unwitting crystallization of a contaminant. Although the targets were ultimately of low importance for the structural insights that they provided, their solution prevented further misdirected effort on the part of the researchers involved. All cases involve the crystallization of a known contaminant. Examples involving the use of $S I M B A D$ for novel structure solution are available elsewhere, such as PDB entries 6byq, $6 c 87$ and 5wol. Cases illustrating the use of SIMBAD for targets that had not been previously sequenced are not shown owing to the publications being in progress at the time of writing. Solutions for mislabelled crystals are also not shown. These cases were of little interest to the researcher once the mistake had been realized, and no further effort was devoted to structure completion.

3.2.1. Escherichia coli DPS protein contaminant. Crystals of the contaminant protein DPS (DNA-protecting protein during starvation) grew in previously established conditions for caspase 1: the vapour-diffusion method with a well solution consisting of $0.1 M$ sodium chloride, $0.1 M$ bis-tris $\mathrm{pH}$ 6.5, 1.5 $M$ ammonium sulfate and hanging drops composed of a 1:1 mixture of the well solution and $8 \mathrm{mg} \mathrm{ml}^{-1}$ protein in a buffer consisting of $50 \mathrm{~m} M$ sodium acetate $\mathrm{pH} 5.9,100 \mathrm{~m} M \mathrm{NaCl}, 5 \%$ glycerol (R. Wu, unpublished results). Crystals did not grow in the expected time range, but appeared after several months at ambient temperature. They were cryoprotected by the addition of $20 \%$ glycerol to the well solution and cryocooled in liquid nitrogen. The crystals belonged to space group $C 222_{1}$, with lattice parameters $a=117.62, b=133.97, c=139.11 \AA$, $\alpha=\beta=\gamma=90^{\circ}$ and a presumed six molecules of caspase 1 in the asymmetric unit. Diffraction data were measured at $100 \mathrm{~K}$ using a PILATUS3 6M detector (Dectris) on the 23ID-D beamline of GMCA@APS at the Advanced Photon Source, Argonne National Laboratory, USA. The data were indexed, integrated and reduced with $X D S$ (Kabsch, 2010).

The SIMBAD MoRDa DB search led to success with the structure of a 167-residue protein identified as a DNAprotecting protein during starvation from E. coli (PDB entry $1 \mathrm{f30}$ ), which is characterized as a ferritin-like protein in the SCOP database (Murzin et al., 1995). After refinement, it became clear that this was the protein that had crystallized instead of caspase 1 . The structure of DPS was refined with REFMAC5 in the CCP4 suite to $1.5 \AA$ resolution, resulting in $R$ and $R_{\text {free }}$ values of $17.64 \%$ and $20.77 \%$, respectively. Manual model inspection and modifications were performed with Coot. In the crystal, 12 molecules of the protein form a hollow sphere closely reminiscent of that formed in crystals of ferritin (Fig. 4). The coordinates and structure factors have been deposited in the Protein Data Bank with accession code 6b0d and the raw data have been deposited in SBGrid (Morin et al., 2013).

Caspase 1 had previously been successfully purified and crystallized, and its structure had been solved using MR (R. Wu, unpublished results). While there were telltale signs of possible contamination of the new protein preparation, they were not clear enough or had plausible alternative explanations. For example, the crystals from the current protein sample looked different from those used in the structure solution of caspase 1 and had very different unit-cell parameters. However, this was attributed to the fact that caspase 1 was cross-linked in the current sample. It was considered possible that the cross-linking might have interfered with the proper folding, since caspase 1 folds from two peptides in a two-step process. Therefore, it was thought that perhaps the final product was structurally significantly different from the molecule whose structure was solved previously. Initial difficulties in MR were attributed to the same possibility.

3.2.2. Serratia proteamaculans cyanase protein contaminant. Crystals of the contaminant protein (cyanase) grew in conditions expected to crystallize a cytokine complex: the vapour-diffusion method with a well solution consisting of $0.1 M$ magnesium acetate, $10 \%$ PEG 10K, $0.1 M$ MES pH 6.5 and hanging drops composed of a 1:1 mixture of the well solution and the protein complex. Crystals appeared after six months at ambient temperature. The crystals were cryoprotected with $20 \%$ ethylene glycol. The crystals belonged to space group $C 121$, with lattice parameters $a=136.56, b=94.13$, $c=89.11 \AA, \alpha=90, \beta=125.49, \gamma=90^{\circ}$ and five molecules in the asymmetric unit. Diffraction data were collected using an ADSC Q315 detector on the MX2 beamline at the Australian Synchrotron. The data were indexed, integrated and reduced with $X D S$.

The SIMBAD MoRDa DB search led to successful structure solution with the 156-residue cyanase from $S$. proteamaculans (PDB entry 4y42). After refinement it became clear that this was the protein that had crystallized instead of the cytokine complex.

The structure of the cyanase was refined with phenix.refine (Adams et al., 2010) to $1.91 \AA$ resolution, yielding $R$ and $R_{\text {free }}$ values of $16.0 \%$ and $20.2 \%$, respectively. Manual model inspection and modifications were performed with Coot. In

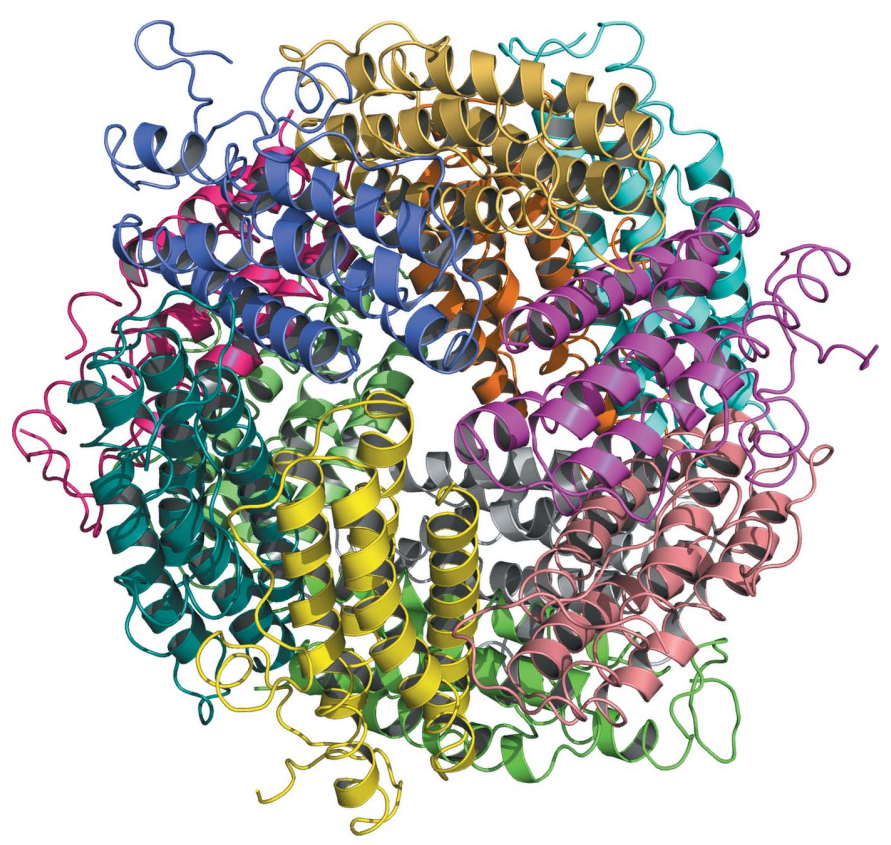

Figure 4

Cartoon representation of the E. coli DPS dodecamer, with protomers identified by colour. 
the crystal, ten molecules of the protein form a dimeric pentagonal ring (Fig. 5). The coordinates and structure factors have been deposited in the Protein Data Bank as entry 6b6m.

Following refinement, the cyanase crystallized was found to have the same sequence as PDB entry $4 y 42$ in spite of the fact that the cytokine was produced in an E. coli cell line and the receptors were produced in insect cells. This suggests that one of the expression organisms had became contaminated with $S$. proteamaculans, which in turn led to the contaminant crystals.

Both the SIMBAD contaminant search and the ContaMiner contaminant search allow users to limit the search to common contaminants from a specific host organism. Normally, this is a logical step that saves computing time; however, this case demonstrates the value of making no assumptions where contaminant origin is concerned.

This case also highlighted a limitation in the iteration of the SIMBAD lattice-parameter search used. PDB entry $4 \mathrm{y} 42$ had been identified as a search model by the lattice-parameter search. However, subsequent MR/refinement failed to provide a solution. Analysis of why PDB entry $4 \mathrm{y} 42$ failed to provide a solution at the lattice-parameter stage revealed an oversight in how structures were being input as search models. At the time that this case was run, all models identified by the latticeparameter search were input into MR with no modifications following download from the PDB. This method had proved sufficient to solve structures which had been crystallized in identical forms to structures already present in the PDB. However, this would break in scenarios where structures were crystallized in symmetry-related space groups.

In this instance, our search model (PDB entry 4y42) was crystallized in space group $P 1$ with ten molecules in the

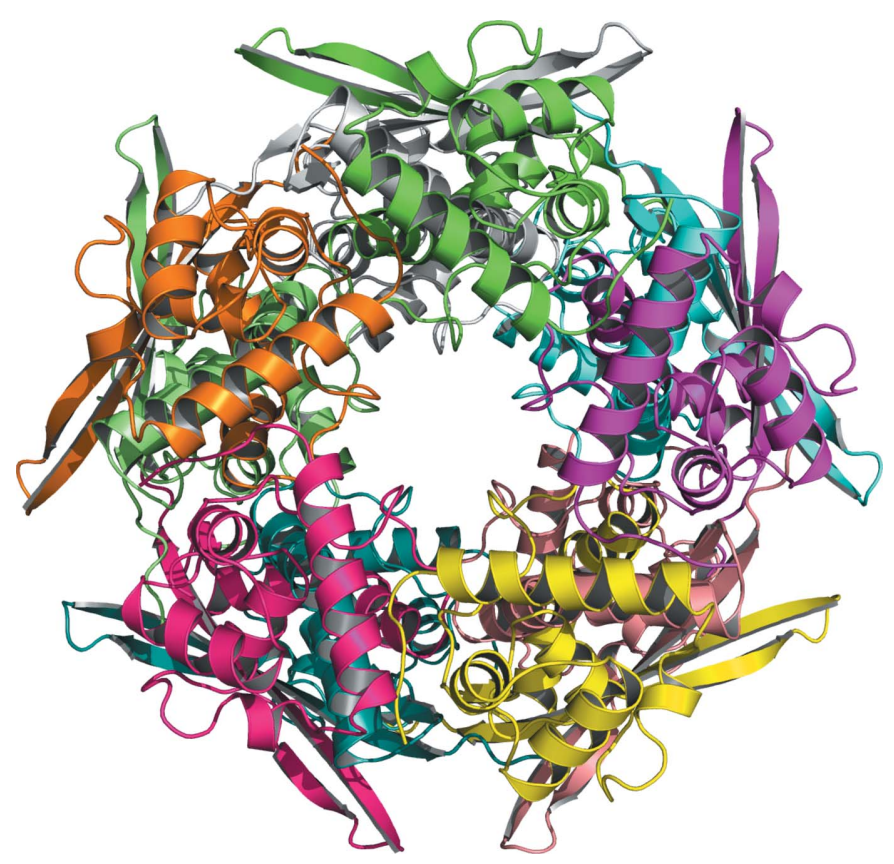

Figure 5

Cartoon representation of the $S$. proteamaculans cyanase decamer, with protomers identified by colour. asymmetric unit, whereas our crystals had crystallized in space group $C 121$ with only five molecules in the asymmetric unit. Using PDB entry $4 \mathrm{y} 42$ as a search model without modification led to the failure of MR as the MR search was trying to place too many monomers. SIMBAD has subsequently been modified as a result of this case to use a Matthews coefficient to check whether a search model can fit into the asymmetric unit prior to MR. If the full PDB entry is too large to be used as a search model, only the first chain is used. This alteration allowed a solution to be found at the lattice-parameter search instead of the MoRDa DB search in subsequent testing.

3.2.3. E. coli catalase HPII protein contaminant. Crystals of the contaminant protein catalase HPII grew from a $10 \mathrm{mg} \mathrm{ml}^{-1}$ solution of target protein A (Fig. 6a). Protein A was produced in E. coli $\mathrm{TOP} 10 \mathrm{~F}^{\prime}$ cells, overexpressed as a recombinant fusion with a $6 \times$ His tag and purified by successive metal-affinity and size-exclusion chromatography steps. Mass spectrometry (4800 MALDI-TOF/TOF, Abi Sciex) confirmed the anticipated identity of the purified target protein. Crystals were thereafter obtained by vapour diffusion after three months of incubation at $19^{\circ} \mathrm{C}$ in $600 \mathrm{nl}$ drops composed of a 1:1 mixture of protein and reservoir solutions in a sitting-drop setup with $90 \mu \mathrm{l}$ reservoir solution $[0.085 \mathrm{M} \mathrm{Na}$ HEPES pH 7.5, 17\%(w/v) PEG 4000, 15\%(v/v) glycerol, $8.5 \%(v / v)$ 2-propanol or $0.1 M$ HEPES pH 7.0, $20 \%(w / v)$ PEG 6000, $1.0 M$ lithium chloride] in the reservoir. Single crystals reached a length of approximately $60 \mu \mathrm{m}$ and were flash-cooled in liquid nitrogen. X-ray diffraction data were collected on beamline I04 at Diamond Light Source, UK employing radiation of wavelength $0.97946 \AA$. The diffraction data were processed using $X D S$ and scaled with AIMLESS (Evans \& Murshudov, 2013) from the CCP4 program suite. The crystals grew in space group $P 1$, with unit-cell parameters $a=69.34, b=90.14, c=114.76 \AA, \alpha=107.10, \beta=105.60$, $\gamma=95.98^{\circ}$, and diffracted X-rays to $2.93 \AA$ resolution. Initial phasing attempts failed using molecular replacement (MR) with models displaying $20-30 \%$ sequence identity to our target (the best hits available in the PDB) as search probes. $A b$ initio/MR phasing strategies such as ARCIMBOLDO also proved to be unsuccessful, likely owing to limited resolution. We decided to optimize the crystallization conditions with the aim of obtaining crystals that diffracted X-rays to higher resolution, as well as to apply experimental phasing methods. Similar crystals did grow in the optimization plates after threemonth incubations from $2 \mu \mathrm{l}$ hanging drops with $1 \mathrm{ml}$ reservoir solution in the reservoir, indicating that crystallogenesis was reproducible, even though new protein batches were used. However, a contaminant search performed at this point with $S I M B A D$ readily identified PDB entry $3 \mathrm{vu} 3$ (Yonekura et al., 2013) as a successful MR search model. Four copies of the $84 \mathrm{kDa}$ product of the E. coli katE gene were found in the $P 1$ unit cell, revealing the known homotetrameric assembly of catalase HPII (Fig. 6b). The structure was refined by iterative cycles of manual model building with Coot and refinement with BUSTER (Bricogne et al., 2017), leading to final $R$ and $R_{\text {free }}$ values of 0.183 and 0.236 , respectively. Atomic coordi- 


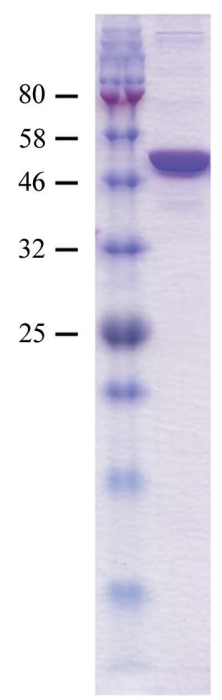

(a)

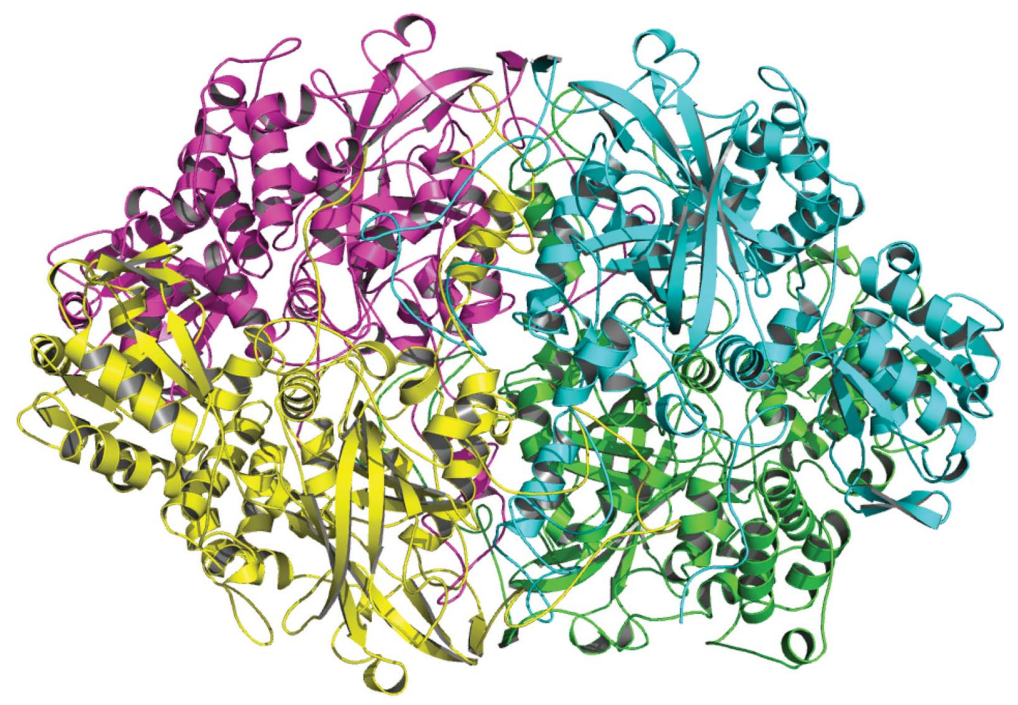

(b)

Figure 6

(a) SDS-PAGE of the protein sample employed for crystallogenesis. Molecular-mass markers are labelled in kDa. (b) Cartoon representation of the $E$. coli catalase HPII tetramer, with protomers identified by colour.

nates and structure factors were deposited in the PDB as entry 6 by0 and the raw data have been deposited in SBGrid. Mass spectrometry with a Quadrupole-Orbitrap hybrid mass spectrometer (Q-Exactive Plus, Thermo) revealed that the E. coli catalase HPII was present in an $\sim 1: 40$ ratio relative to our target in the protein samples employed for crystallization. Even though catalase HPII is a known contaminant that is prone to crystallize (Yonekura et al., 2013), the $P 1$ crystal lattice had not previously been reported, escaping a PDB-wide search as demonstrated by the $S I M B A D$ lattice-parameter search.

\section{Discussion}

$S I M B A D$ has been designed to be used in a range of different scenarios where conventional sequence-based MR methods have failed. So far, SIMBAD has proved to be effective at identifying crystal contaminants, as also have other similar methods such as MarathonMR (Hatti et al., 2016) and ContaMiner (Hungler et al., 2016), suggesting that contamination is one of the main reasons that conventional methods fail. Alongside MarathonMR (Hatti et al., 2017), SIMBAD has also proved effective in cases where crystals have been mislabelled. This can happen for various reasons, especially in multi-laboratory collaborations. SIMBAD has also successfully determined the structures of unsequenced proteins and a case of swapped crystallization trays (data not shown). More ambitiously, SIMBAD also provides a possible means to solve a novel target which is structurally similar to an existing protein in the MoRDa DB but whose relationship to that structure is not apparent by sequence comparisons alone.

The different elements of the $S I M B A D$ pipeline have very different computational demands. The fastest step in the pipeline is the lattice-parameter search. The experimental lattice parameters are compared with the lattice parameters stored in the Niggli cell database (129947 at the time of writing) in less than $10 \mathrm{~s}$. Subsequent MR can take as little as $30 \mathrm{~s}$ when the top-scoring search model results in a solution and typically less than 15 min for more difficult cases. The next fastest step, the contaminant search, typically runs in about 15 min using four cores $(3.2 \mathrm{GHz}$, Intel i5-6500) when run against the full contaminant database (349 structures and 443 associated $M o R D a$ domains at the time of writing). Users can reduce the number of search models to try by specifying the expression organism using the UniProt mnemonic (The UniProt Consortium, 2017); for example, E. coli would be ECOLI whereas Saccharomyces cerevisiae (strain ATCC 204508/S288c) would be YEAST. This can improve the speed of the contaminant search, although it could also reduce its effectiveness in cases where the expression organism cell line has become contaminated by a different microorganism. The lattice-parameter and contaminant searches are very quick, and could easily be run routinely after data collection on beamlines to check for the possibility of a contaminant/ mislabelled protein. This would allow the identification of a problem and suggest additional data collection from a different crystallization trial when available.

The most time-consuming step in the pipeline is the MoRDa DB search. Using a 100-core cluster $(2.8 \mathrm{GHz}$, AMD Opteron 4184) on cases where all 90000 search models were tried, the MoRDa DB search typically took 4-12 h. When fewer than 90000 search models were tried, the MoRDa DB search was significantly quicker. For example, using the 100-core cluster on TOXD (a 59-amino-acid $\alpha$-dendrotoxin with one molecule in the asymmetric unit that is distributed as an example case by CCP4) took less than an hour as only 20000 search models could potentially fit into the unit cell. Whereas the lattice-parameter search and contaminant search are suitable for use on desktop computers, the MoRDa DB search is primarily aimed at clusters. Nonetheless, testing has found that 
the MoRDa DB search can also be run tolerably quickly on a modern multi-core desktop. Using an eight-core/16-thread machine $(3.0 \mathrm{GHz}$, Intel $\mathrm{i} 7-5960 \mathrm{X})$, the MoRDa DB search took between 1 and $2 \mathrm{~d}$ on a range of test cases where no search models were excluded. The MoRDa DB itself requires $2.8 \mathrm{~GB}$ of storage.

\subsection{Future developments}

There are several areas that will be explored in the future to determine whether they improve the effectiveness of $S I M B A D$. A key area will be expanding the database used by the MoRDa DB search to also include truncated variants and oligomeric forms of proteins. As the MoRDa DB is a reduced database, the top model identified by the SIMBAD MoRDa DB search will not necessarily be the closest available match in the PDB. Therefore, another area to explore is whether homologues of the best search model which were removed when the redundancy was reduced in the construction of the MoRDa DB can provide a better MR solution.

To date, it has been difficult to build an accurate picture of common contaminants, as these structures often go either unsolved or unpublished.

As SIMBAD becomes used more regularly we foresee the possibility of gathering significantly more data on common contaminants and therefore improving our contaminant database. We are also developing SIMBAD to use ContaBase (provided by ContaMiner) as a source to update our contaminant database. Therefore, in the event that a user identifies a novel contaminant, we suggest submitting the contaminant to ContaBase, where it will benefit both future SIMBAD and ContaMiner searches.

Another avenue to explore is whether alternative scoring systems increase the effectiveness of SIMBAD, as might alternative MR programs for the rotation search in place of the current Patterson-based AMoRe search. In particular, we plan to explore the maximum-likelihood-based rotation search in Phaser using its convenient capacity to process a batch of search models in a single job. Of key interest will be how other MR programs affect the sensitivity of the pipeline and its speed.

Currently, the lattice-parameter search and contaminant search are available in $C C P 4 i$ and $C C P 4 i 2$ on *nix-based architectures, with plans to bring SIMBAD to CCP4 online services.

\section{Conclusions}

Crystal contamination is a possibility that every crystallographer should bear in mind when performing an experiment. $S I M B A D$ provides a rapid and reliable means to check for the presence of a contaminant. SIMBAD is also useful in cases of the misidentification of a crystal and can also be useful in scenarios where no obvious homologue is available as a search model or the most suitable search model is not among those most highly ranked by sequence comparisons. The latticeparameter and contaminant searches in SIMBAD are very quick, and we therefore suggest running them routinely after data collection on beamlines to identify possible cases of contaminant crystallization or protein mislabelling.

\section{Acknowledgements}

The development of SIMBAD was inspired by a collaboration with Jon Cooper of UCL. We would also like to thank Martyn Winn for his useful suggestions on the development of the $S I M B A D$ pipeline. We acknowledge the part of the work performed on the DPS protein at the CCP4/APS school in 2016. RW thanks Gregory A. Petsko from Weill Cornell Medicine for support of the research.

\section{Funding information}

The development of SIMBAD has been supported by the BBSRC CCP4 grant BB/L009544/1, the University of Liverpool and Synchrotron SOLEIL. GM/CA@APS has been funded in whole or in part with Federal funds from the National Cancer Institute (ACB-12002) and the National Institute of General Medical Sciences (AGM-12006). This research used resources of the Advanced Photon Source, a US Department of Energy (DOE) Office of Science User Facility operated for the DOE Office of Science by Argonne National Laboratory under Contract No. DE-AC02-06CH11357. The work on cytokine/cyanase was supported by grant APP1004945 from the Australian National Health and Medical Research Council (NHMRC) and was made possible at WEHI through Victorian State Government Operational Infrastructure Support and the Australian NHMRC Independent Research Institutes Infrastructure Support Scheme. M-NL received a postdoctoral fellowship from the ANII (Agencia Nacional de Investigación e Innovación, Uruguay).

\section{References}

Adams, P. D. et al. (2010). Acta Cryst. D66, 213-221.

Andrews, L. C. \& Bernstein, H. J. (2014). J. Appl. Cryst. 47, 346-359.

Bibby, J., Keegan, R. M., Mayans, O., Winn, M. D. \& Rigden, D. J. (2012). Acta Cryst. D68, 1622-1631.

Bricogne, G., Blanc, E., Brandl, M., Flensburg, C., Keller, P., Paciorek, W., Roversi, P., Sharff, A., Smart, O. S., Vonrhein, C. \& Womack, T. O. (2017). BUSTER v.2.10.3. Global Phasing Ltd., Cambridge, UK.

Caliandro, R., Carrozzini, B., Cascarano, G. L., Giacovazzo, C., Mazzone, A. \& Siliqi, D. (2009). Acta Cryst. A65, 512-527.

Crowther, R. A. (1972). The Molecular Replacement Method, edited by M. G. Rossmann, pp. 173-178. New York: Gordon \& Breach.

Crowther, R. A. \& Blow, D. M. (1967). Acta Cryst. 23, 544-548.

Emsley, P., Lohkamp, B., Scott, W. G. \& Cowtan, K. (2010). Acta Cryst. D66, 486-501.

Evans, P. \& McCoy, A. (2008). Acta Cryst. D64, 1-10.

Evans, P. R. \& Murshudov, G. N. (2013). Acta Cryst. D69, 1204-1214.

Foadi, J., Woolfson, M. M., Dodson, E. J., Wilson, K. S., Jia-xing, Y. \& Chao-de, Z. (2000). Acta Cryst. D56, 1137-1147.

Hatti, K., Biswas, A., Chaudhary, S., Dadireddy, V., Sekar, K., Srinivasan, N. \& Murthy, M. R. N. (2017). J. Struct. Biol. 197, 372378.

Hatti, K., Gulati, A., Srinivasan, N. \& Murthy, M. R. N. (2016). Acta Cryst. D72, 1081-1089.

Hoppe, W. (1957). Angew. Chem. 69, 659-674.

Huber, R. (1965). Acta Cryst. 19, 353-356. 
Hungler, A., Momin, A., Diederichs, K. \& Arold, S. T. (2016). J. Appl. Cryst. 49, 2252-2258.

Jenkins, H. T. (2018). Acta Cryst. D74, 205-214.

Kabsch, W. (2010). Acta Cryst. D66, 125-132.

Keegan, R. M., McNicholas, S. J., Thomas, J. M. H., Simpkin, A. J., Simkovic, F., Uski, V., Ballard, C. C., Winn, M. D., Wilson, K. S. \& Rigden, D. J. (2018). Acta Cryst. D74, 167-182.

Keegan, R., Waterman, D. G., Hopper, D. J., Coates, L., Taylor, G., Guo, J., Coker, A. R., Erskine, P. T., Wood, S. P. \& Cooper, J. B. (2016). Acta Cryst. D72, 933-943.

Krissinel, E. (2007). Bioinformatics, 23, 717-723.

Krissinel, E. (2012). J. Mol. Biochem. 1, 76-85.

Krissinel, E. \& Henrick, K. (2004). Acta Cryst. D60, 2256-2268.

Krissinel, E., Uski, V., Lebedev, A., Winn, M. \& Ballard, C. (2018). Acta Cryst. D74, 143-151.

Long, F., Vagin, A. A., Young, P. \& Murshudov, G. N. (2008). Acta Cryst. D64, 125-132.

McCoy, A. J., Grosse-Kunstleve, R. W., Adams, P. D., Winn, M. D., Storoni, L. C. \& Read, R. J. (2007). J. Appl. Cryst. 40, 658-674.

McCoy, A. J., Oeffner, R. D., Wrobel, A. G., Ojala, J. R. M., Tryggvason, K., Lohkamp, B. \& Read, R. J. (2017). Proc. Natl Acad. Sci. USA, 114, 3637-3641.

McGill, K. J., Asadi, M., Karakasheva, M. T., Andrews, L. C. \& Bernstein, H. J. (2014). J. Appl. Cryst. 47, 360-364.

Morin, A., Eisenbraun, B., Key, J., Sanschagrin, P. C., Timony, M. A., Ottaviano, M. \& Sliz, P. (2013). Elife, 2, e01456.

Murshudov, G. N., Skubák, P., Lebedev, A. A., Pannu, N. S., Steiner, R. A., Nicholls, R. A., Winn, M. D., Long, F. \& Vagin, A. A. (2011). Acta Cryst. D67, 355-367.

Murzin, A. G., Brenner, S. E., Hubbard, T. \& Chothia, C. (1995). J. Mol. Biol. 247, 536-540.

Navaza, J. (1987). Acta Cryst. A43, 645-653.
Navaza, J. (1993). Acta Cryst. D49, 588-591.

Navaza, J. (1994). Acta Cryst. A50, 157-163.

Niedzialkowska, E., Gasiorowska, O., Handing, K. B., Majorek, K. A., Porebski, P. J., Shabalin, I. G., Zasadzinska, E., Cymborowski, M. \& Minor, W. (2016). Protein Sci. 25, 720-733.

Qian, B., Raman, S., Das, R., Bradley, P., McCoy, A. J., Read, R. J. \& Baker, D. (2007). Nature (London), 450, 259-264.

Ramraj, V., Evans, G., Diprose, J. M. \& Esnouf, R. M. (2012). Acta Cryst. D68, 1697-1700.

Read, R. J. (1999). Acta Cryst. D55, 1759-1764.

Read, R. J. (2001). Acta Cryst. D57, 1373-1382.

Rigden, D. J., Keegan, R. M. \& Winn, M. D. (2008). Acta Cryst. D64, 1288-1291.

Rodríguez, D. D., Grosse, C., Himmel, S., González, C., de Ilarduya, I. M., Becker, S., Sheldrick, G. M. \& Usón, I. (2009). Nature Methods, 6, 651-653.

Rossmann, M. G. \& Blow, D. M. (1962). Acta Cryst. 15, $24-31$.

Sharma, H., Yu, S., Kong, J., Wang, J. \& Steitz, T. A. (2009). Proc. Natl Acad. Sci. USA, 106, 16604-16609.

Stokes-Rees, I. \& Sliz, P. (2010). Proc. Natl Acad. Sci. USA, 107, 21476-21481.

Storoni, L. C., McCoy, A. J. \& Read, R. J. (2004). Acta Cryst. D60, 432-438.

The UniProt Consortium (2017). Nucleic Acids Res. 45, D158-D169.

Thorn, A. \& Sheldrick, G. M. (2013). Acta Cryst. D69, 2251-2256.

Vagin, A. \& Lebedev, A. (2015). Acta Cryst. A71, s19.

Vagin, A. \& Teplyakov, A. (2010). Acta Cryst. D66, 22-25.

Winn, M. D. et al. (2011). Acta Cryst. D67, 235-242.

Yao, J., Woolfson, M. M., Wilson, K. S. \& Dodson, E. J. (2005). Acta Cryst. D61, 1465-1475.

Yonekura, K., Watanabe, M., Kageyama, Y., Hirata, K., Yamamoto, M. \& Maki-Yonekura, S. (2013). PLoS One, 8, e78216. 